\title{
Requirements for a Nutrition Education Demonstrator ${ }^{f}$
}

\author{
Ing Widya ${ }^{1}$, Richard Bults ${ }^{1}$, Rene de Wijk ${ }^{2}$, Ben Loke $^{3}$, Nicole Koenderink ${ }^{2}$, \\ Ricardo Batista $^{1}$, Val Jones ${ }^{1}$, Hermie Hermens ${ }^{1}$ \\ ${ }^{1}$ Remote Monitoring \& Treatment group, University of Twente, the Netherlands, \\ ${ }^{2}$ Wageningen University \& Research, Food \& Biobased Research, the Netherlands, \\ ${ }^{3}$ Noldus Information Technology BV, the Netherlands, \\ widya@ewi.utwente.nl
}

\begin{abstract}
Context and Motivation] Development of innovative ICT-based applications is a complex process involving collaboration of all relevant disciplines. This complexity arises due to differences in terminology, knowledge and often also the ways of working between developers in the disciplines involved. [Question/problem] Advances in each discipline bring a rich design environment of theories, models, methods and techniques. Making a selection from these makes the development of distributed applications very challenging, often requiring a holistic approach to address the needs of the disciplines involved. This paper describes early stage requirements acquisition of a mobile nutrition education demonstrator which supports overweight persons in adopting healthier dietary behaviour. [Principal idea/results] We present a novel way to combine and use known requirements acquisition methods involving a two stage user needs analysis based on scenarios which apply a theory-based model of behavioural change and are constructed in two phases. The first phase scenarios specify an indicative description reflecting the use of the transtheoretical model of behavioural change. In the second phase, a handshake protocol adds elements of optative system-oriented descriptions to the scenarios such that the intended system can support the indicative description. [Contribution] The holistic and phased approach separates design concerns to which each of the disciplines contributes with their own expertise and domain principles. It preserves the applied domain principles in the design and it bridges gaps in terminology, knowledge and ways of working.
\end{abstract}

Key words: requirements acquisition, nutrition education, scenario-based design, transtheoretical model of behavioural change.

\section{Introduction}

Exposure to plentifully available food in today's $24 / 7$ economy, together with our increasingly sedentary behaviour, are blamed for causing an epidemic of overweight and obesity in many countries [1]. In this context, nutrition education is receiving increasing attention in the drive to improve public health. We define nutrition

${ }^{f}$ This work is funded by the Dutch Ministry of Economic Affairs \& the Province of Gelderland under Pilot Gebiedsbericht Innovatiebeleid programme in FOVEA (www.project-fovea.org). 
education as "any combination of educational strategies, accompanied by environmental supports, designed to facilitate voluntary adoption of food choices and other food- and nutrition-related behaviors conducive to health" ([2], p. 15). Studies report that nutrition education interventions which aim at behavioural change have significant effect in improving dietary practices [2].

One challenge is to investigate how Information and Communication Technology (ICT) based systems can facilitate nutrition education processes by re-engineering the environment of individuals to support them in dealing with unhealthy food temptations. In the multidisciplinary FOVEA project, food scientists and ICT engineers collaborate in the development of a mobile peer-to-peer demonstrator which facilitates overweight persons in adopting healthier dietary behaviour by making healthy food choices which balance energy intake and energy expenditure.

This paper reports on the early stage requirements acquisition of the demonstrator; later stage requirements related to common look and feel of device interfaces are not in the scope of this paper. To our knowledge, we present a novel way to combine and use known requirements acquisition methods in a collaboration process between the disciplines. The process specifies scenarios in two phases such that the acquired requirements align to the application's underlying principles and are feasible to implement.

This work has been inspired by requirements engineering and nutrition education literature. Scenario-based user needs analysis has been discussed in many articles, including [3, 4]. Development of scenarios that reflect medical guidelines and envisioned healthcare interventions has been proposed in [5]. Nutrition education interventions often adopt healthcare models. In this domain, the determinants and need for a healthier food choice and the relevance of behaviour change theories and models (e.g. [6]) have been discussed in [1, 2].

\section{Nutrition Education Intervention}

This section discusses food choice factors and a model for behavioural change.

Food factors. Many factors influence food choices and dietary behaviour of individuals; for example, biologically determined preferences such as sensory factors (e.g. sweet, salt and hunger), experience or familiarity with food, cultural norms and individual beliefs (e.g. green is healthy), environmental factors (e.g. availability and price) or social structure influences [2]. Food choice therefore depends on the individual, but some influencing factors are common to a community. Dietary strategies may make use of these common factors to manage desirable food choices but need also to take account of the individual's food factor profile, for example for weighing risk and enjoyment of unhealthy food.

Our work uses food factors to specify levels of unhealthy food temptations so as to instrument the demonstrator with ICT-support that engineers an augmented reality environment such that individuals can better deal with these temptations. 
Transtheoretical model. Nutrition education adopts socio-psychological models of behavioural change. The transtheoretical model (TTM) is an intentional change model focusing on decisions of the individual [6]. In this model, behavioural change occurs gradually and dynamically throughout a series of five stages: Precontemplation (individuals are not interested in changing behaviour), Contemplation (individuals are considering changing behaviour but weigh the cost and benefit which often proves a barrier to progress to the next stage), Preparation (individuals are motivated and intend to change), Action (individuals have taken first steps to change; e.g. have learned to cope with some food cues but need action-oriented support to improve self efficacy), Maintenance (individuals adopt a new learned behaviour but have to exert effort to prevent relapse).

Typical in TTM is that factual and procedural knowledge necessary for change are considered insufficient for individuals in the preparation stage to take action to change their behaviour. These individuals need support to set action plans and goals. They have to be facilitated to make deliberate decisions, enabling them to weigh benefits and effort of intended actions. These supports bridge the intention to action gap between the preparation and action stages. Support for self-efficacy is furthermore essential in behavioural models in general.

TTM identifies ten mechanisms enabling individuals to progress through stages, for example stimulus control (to manage unhealthy food cues) and helping relationships (to get external help to change).

Feedback mechanisms. Feedback is an important instrument in nutrition education to facilitate self-efficacy. Feedback may be used to advise individuals to avoid unhealthy food cues (cue avoidance) or to notify them for cues to be managed (cue control).

Feedback is also needed to improve awareness of eating (mindful eating) to reduce the risk of overeating. In mindful eating, feedback can be used to slow down the speed of eating such that food intake is better synchronized with the delayed feeling of satiation. Feedback types (e.g. positive or negative), modalities, frequency and content depend on the context and objective of the feedback.

\section{Requirements Acquisition Methodology}

The early stage requirements acquisition methodology applies a waterfall model which consists of two stages; the first stage consists of two phases.

In the first phase, domain experts bring in their theories, models or guidelines into the envisioned intervention specified as an indicative description by scenarios $[4,5$, 7]. These scenarios are expressed in terms of PACT [8], which stands for People (i.e. actors in the scenario), Activities (e.g. intervention relevant activities of the actors), Context (i.e. the environmental circumstance of the actors and activities) and Technologies (i.e. essential technologies for the intervention).

Activities specified in the PACT scenarios may be expressed independently from the need for ICT support or the way the intended system eventually mediates those of them which do require ICT support. An analysis of the activities which require ICT support determines the required functionality of the intended system. 
In the second phase, ICT engineers with experience in systems development propose to augment the PACT scenarios with FICS elements [8], including the usersystem interactions needed to realize PACT activities that need ICT support. Adapted to our nutrition education context, FICS stands for Functions (i.e. functionality of the intended system which is capable to realize actor's activities), Interactions (i.e. usersystem or system-component interactions mediating actor's activities), Content (i.e. variables of the interactions) and Services (i.e. types of the interactions, e.g. unidirectional data streaming service or reliable messaging service). FICS elements describe how the intended system mediates actors' activities and therefore form an optative (i.e. wished) service description of the intended system [7].

If the domain experts believe that the proposed interactions indeed mediate the PACT activities and are aligned with their intentions, they acknowledge the proposal and augment the PACT scenarios with these interactions, resulting in combined PACT-FICS scenarios. This procedure therefore implements a two-way handshake communication protocol in which scenarios become the common discourse of the collaborating disciplines; therefore, reducing the risk of misinterpretation.

In the second stage, the ICT engineers become the lead designers and further analyze user needs given the agreed-upon PACT-FICS scenarios. These engineers may apply requirements engineering methods, techniques and tools without preoccupation of application domain theories or models applied.

Augmented methods. Cross-disciplinary studies further reduce the risk of misinterpretation mentioned earlier; so the ICT engineers studied literature on behavioural change models and nutrition education. The knowledge gained made collaboration in a multidisciplinary setting easier and more effective. Task analysis was used to complement the scenario development and analysis. Goal analysis may also reveal design alternatives, but was marginally used in the current exercise.

\section{Development trajectory}

This section addresses some of the design choices, rationale and results of the use of the methodology in the development of the nutrition education demonstrator.

Design choices and rationale. The envisioned nutrition education intervention applies TTM to change the dietary behaviour of an overweight inclined abstainer who also is typified as an external eater. An inclined abstainer knows the risk of unhealthy food choices and is motivated to adopt a healthier dietary behaviour, but experiences difficulties in moving forward from having intentions to taking action. As an external eater, this abstainer is easily tempted by external unhealthy food cues; therefore, progresses and relapses between the TTM preparation, action and maintenance stages. An augmented reality environment re-engineered by means of a mobile nutrition education demonstrator may help this abstainer to control unhealthy food cues.

PACT scenarios are considered suitable to express the envisioned nutrition education intervention. They specify activities of the abstainer (People and Activities) 
to manage external food cues (Context) by using a mobile application (Technology). Thereafter, the implementation aspects can be addressed using FICS elements.

Results. Dietary behavioural interventions are not only based on food choice control at eating moments. Energy intake and expenditure need to be balanced over longer periods; for example at a daily or weekly basis. Accordingly, a comprehensive set of scenarios which cover daily and weekly activities were developed under responsibility of the food scientists in the project. These scenarios result in eighteen small PACT and FICS requirements tables specifying indicative activities (i.e. not all activities need to be mediated by the intended system) and optative interactions (i.e. required to realize the activities which need the system's mediation), respectively. Besides activities or interactions, the tables describe the actors, the intentions or goals (e.g. cue control or avoidance), and the functionality or resource components needed.

The lunch scenario of the abstainer at the Restaurant of the Future (RoF) [9] was selected for further elaboration towards the demonstrator. The RoF is a company restaurant which also acts as a field laboratory, enabling close observation of people during selection of food items and during eating and drinking. Extensive discussions and five iterations result in a detailed lunch scenario which complies with the comprehensive scenarios. This scenario focuses on food choice aspects as well as mindful eating aspects. Moreover, it incorporates both cue avoidance and cue control.

A time-sequence diagram of interactions of the abstainer with the intended system has been derived from the lunch scenario. From this diagram, a state diagram of usersystem and system component interactions has been derived and expressed in UML. Further, an associated service-oriented state diagram has been specified describing state change conditions and the interactions observable at the user-system interface of the mobile component of the intended system. This last mentioned state diagram is expressed by a screen mock-up for abstainer's mobile device, which acts as input device of the abstainer and which outputs informative or feedback actions initiated internally by the mobile component or externally by the RoF's peer component.

\section{Discussion and Conclusions}

This paper discusses the acquisition of requirements of a dietary intervention for supporting healthier behaviour, despite external temptations of unhealthy food, implemented as a mobile demonstrator. The acquisition process followed known pathways of requirements engineering once the combined PACT-FICS scenarios have been derived. A task analysis complemented the acquisition process further. The elaborated lunch scenario and the screen mock-up, representing the service level state diagram at the mobile device, have been validated by food scientists and were presented at a stakeholder workshop of the project.

The PACT and FICS notions and their use in the development process were found to separate the application domain from the system support concerns effectively. Under responsibility of the food scientists, the envisioned intervention was defined without much burden of implementation issues. These food scientists have sufficient background in the basic principles which underlie the dietary intervention. ICT 
engineers bring in their system development experience in the second phase. The use of scenarios as a common discourse is considered effective to reduce misinterpretation and was found to bridge the terminology and knowledge gaps between the collaborating disciplines, but the process needed several iterations despite the fact that the ICT engineers studied nutrition education (including TTM).

However, the study may have caused a technological push affecting the first phase PACT scenarios, which turned out to contain more FICS elements than desired. But these FICS elements could have been implied by the focus on cue control which reengineered the abstainer's environment.

Due to pragmatic reasons decided during the second requirements acquisition stage, cue control and avoidance feedback were not implemented directly at RoF food counters but provided indirectly by emoticons on the abstainer's mobile device. This latter is a valid helping relationship in TTM, but from a design perspective it is a design deficiency because the design starting point was to use stimulus control only.

Experience also showed that incorporation of domain principles in scenarios is a necessity, otherwise scenario activities may become aimless. Analysis of such activities does not easily lead to befitting FICS elements or to implementation alternatives. Moreover, incorporation of TTM and feedback in the developed PACT scenarios was not easy due to the state of the art in applying these theories in practice. Furthermore, experience in applying these theories in nutrition education intervention was felt missing in the project. Particularly, a dietician should be involved in the project, amongst others because it was difficult to determine the type of feedback to apply in mindful eating (e.g. positive feedback to acquire the skill or negative feedback for prolonged learning it?). These are topics for future work.

\section{References}

1. Crawford, D., Ball, K.: Behavioural determinants of the obesity epidemic. Asia Pacific J. Clin. Nutr. 11 (Suppl.), S718-721 (2002)

2. Contento, I.R.: Nutrition Educations: Linking Research, Theory and Practice. Jones and Bartlett Publ., Sudbury MA. (2007)

3. Van Helvert, J., Fowler, C.: Scenario-based User Needs Analysis. Chimera Working Paper 2003-02, Colchester, University of Essex (2003)

4. Widya, I., Bults, R.G.A., Huis in 't Veld, R.M.H.A., Vollenbroek-Hutten, M.M.R.: Scenario-based Requirements Elicitation in a Pain-teletreatment Application. In Proc. of the 4th. Int. Conf. on Software and Data Technologies (ICSOFT), 406-413 (2009)

5. Huis in 't Veld, R.M.H.A., Widya, I., Bults, R.G.A., Sandsjö, L., Hermens, H.J., Vollenbroek-Hutten, M.M.R.: A scenario guideline for designing new teletreatments: a multidisciplinary approach. Journal of Telemedicine and Telecare 16, $302-307$ (2010)

6. Velicer, W.F., Prochaska, J.O., Fava, J.L., Norman, G.J., Redding, C.A.: Smoking cessation and stress management: Applications of the Transtheoretical Model of behavior change. Homeostasis 38, 216-233 (1998)

7. Jackson, M.: The meaning of requirements. Ann. of Software Engineering 3, 5-21 (1997)

8. Benyon, D., Macaulay, C.: Scenarios and the HCI-SE design problem. Interacting with Computers 14, 397-405 (2002)

9. Restaurant of the Future, Wageningen UR centre, http://www.restaurantofthefuture.nl 